\title{
SOME EXTREME DISCRETE GROUPS
}

\author{
TROELS JØRGENSEN and MAIRE KIIKKA
}

Let $X$ and $Y$ be two Möbius transformations generating a nonelementary discrete group. A lemma in [2] then tells us that

$$
\left|\tau^{2}(X)-4\right|+\left|\tau\left(X Y X^{-1} Y^{-1}\right)-2\right| \geq 1,
$$

where $X$ and $Y$ are thought of as complex unimodular 2-by-2 matrices (and $X^{-1}$ and $Y^{-1}$ as the inverse matrices) and $\tau$ denotes the trace function.

We shall say that the group generated by $X$ and $Y$ is extreme if equality holds good in (*). In this case it will be proved that $X$ must be elliptic or parabolic and, knowing that, we deduce that the triangle groups of signatures $(2,3, q), q \in\{7,8,9, \ldots, \infty\}$ are the only Fuchsian groups which are extreme.

The following well known identity is useful:

(1) $\tau^{2}(X)+\tau^{2}(Y)+\tau^{2}(X Y)=\tau\left(X Y X^{-1} Y^{-1}\right)+\tau(X) \tau(Y) \tau(X Y)+2$.

We can now establish our first result.

Th e or e m 1. Suppose $X$ and $Y$ generate a non-elementary discrete group and

$$
\left|\tau^{2}(X)-4\right|+\left|\tau\left(X Y X^{-1} Y^{-1}\right)-2\right|=1 .
$$

Then $X$ and $Y_{1}=Y X Y^{-1}$ generate a non-elementary discrete group and

$$
\left|\tau^{2}(X)-4\right|+\left|\tau\left(X Y_{1} X^{-1} Y_{1}^{-1}\right)-2\right|=1 .
$$

Proof. The group generated by $X$ and $Y_{1}$ is discrete because it is a subgroup of the discrete group generated by $X$ and $Y$.

Suppose $X$ is parabolic. Then the group generated by $X$ and $Y_{1}$ were elementary if and only if the fixed point of $X$ were fixed by $Y_{1}$ and hence by $Y$. As the group generated by $X$ and $Y$ is non-elementary, this is not so.

If $\tau\left(X Y X^{-1} Y^{-1}\right)$ were equal to 2 , then $X$ and $Y$ would have 
a common fixed point. This is not so, the group generated by $X$ and $Y$ being non-elementary. Thus $\left|\tau^{2}(X)-4\right|$ is strictly less than 1 , by (2), and hence the order of $X$ exceeds 6 . Therefore, assuming now that $X$ is elliptic or loxodromic, if the group generated by $X$ and $Y_{1}$ were elementary, then either $Y_{1}$ would keep the fixed points of $X$ fixed or $Y_{1}$ would interchange the fixed points of $X$. In the former case we deduce that also $Y$ would either keep the fixed points of $X$ fixed or $Y$ would interchange the fixed points of $X$. In the latter case, $Y$ would have to interchange the fixed points of $X$. None of the two cases can thus occur since the group generated by $X$ and $Y$ is non-elementary.

We have proved that the group generated by $X$ and $Y_{1}$ is nonelementary.

To verify (3), we apply (1) to $X$ and $Y_{1}^{-1}$. Since $X$ and $Y_{1}$ are conjugate we have $\tau(X)=\tau\left(Y_{1}\right)$. Also, it is easy to see that $\tau\left(X Y_{1}^{-1} X^{-1} Y_{1}\right)=\tau\left(X Y_{1} X^{-1} Y_{1}^{-1}\right)$. Thus a short calculation yields

$$
\begin{gathered}
\tau\left(X Y_{1} X^{-1} Y_{1}^{-1}\right)-2 \\
=\left[\tau\left(X Y X^{-1} Y^{-1}\right)-2\right]\left[\tau\left(X Y X^{-1} Y^{-1}\right)-\tau^{2}(X)+2\right] .
\end{gathered}
$$

Because of (2) and the triangle inequality, we get from (4)

$$
\left|\tau\left(X Y_{1} X^{-1} Y_{1}^{-1}\right)-2\right| \leq\left|\tau\left(X Y X^{-1} Y^{-1}\right)-2\right| \text {. }
$$

Applying (2) once more, we get from (5)

$$
\left|\tau^{2}(X)-4\right|+\left|\tau\left(X Y_{1} X^{-1} Y_{1}^{-1}\right)-2\right| \leq 1,
$$

but here equality must hold good since otherwise the discreteness inequality (*) (stated at the beginning) would be violated.

We have proved Theorem 1.

Th e o r e m 2. Suppose $X$ and $Y$ generate a non-elementary discrete group and

$$
\left|\tau^{2}(X)-4\right|+\left|\tau\left(X Y X^{-1} Y^{-1}\right)-2\right|=1 .
$$

Then $X$ is elliptic of order at least 7 or $X$ is parabolic. Furthermore, if $X$ is elliptic, then $\tau\left(X Y X Y^{-1}\right)=1$.

Proof. Suppose that $X$ is not parabolic. Then we have $\tau^{2}(X) \neq 4$. Consider again the element $Y_{1}=Y X Y^{-1}$. As a corollary to the proof of Theorem 1, we have

$$
\begin{gathered}
\left|\tau\left(X Y X^{-1} Y^{-1}\right)-\tau^{2}(X)+2\right| \\
=\left|\tau\left(X Y X^{-1} Y^{-1}\right)-2\right|+\left|\tau^{2}(X)-4\right|,
\end{gathered}
$$

this because (5) was seen to hold good with equality. Consequently, the ratio between $\tau\left(X Y X^{-1} Y^{-1}\right)-2$ and $4-\tau^{2}(X)$ must be a positive 
real number. Notice here that $\tau\left(X Y X^{-1} Y^{-1}\right) \neq 2$ since $X$ and $Y$ generate a non-elementary group.

Repeating the argument, now with $Y_{2}=Y_{1} X Y_{1}^{-1}$ instead of $Y_{1}$, we deduce that $\tau\left(X Y_{1} X^{-1} Y_{1}^{-1}\right)-2$ must be a positive multiple of $4-\tau^{2}(X)$.

The formula (4) then shows that $4-\tau^{2}(X)$ is a positive real number. It is less than 1 because of (2). Hence, $X$ is elliptic of order at least 7 .

Furthermore, we see that $\tau\left(X Y X^{-1} Y^{-1}\right)-2$ is a positive real number. Thus (2) may be written as

$$
\tau\left(X Y X^{-1} Y^{-1}\right)-\tau^{2}(X)+2=1
$$

and since

$$
\tau\left(X Y X^{-1} Y^{-1}\right)+\tau\left(X Y X Y^{-1}\right)=\tau^{2}(X),
$$

also the last assertion in Theorem 2 is proved.

Theorem 3. The only extreme Fuchsian groups are the triangle groups of signatures $(2,3, q)$, where $q$ belongs to $\{7,8,9, \ldots, \infty\}$.

Proof. Let $G$ be an extreme Fuchsian group and $X$ and $Y$ a pair of generators so that (2) is satisfied.

If $X$ is parabolic, then we get from (1)

$$
\tau\left(X Y X^{-1} Y^{-1}\right)-2=(\tau(Y) \pm \tau(X Y))^{2} .
$$

Since $G$ is Fuchsian, all traces are real numbers. Hence, together (2) and (8) give $\tau\left(X Y X^{-1} Y^{-1}\right)=3$. Using (7), we have $\tau\left(X Y X Y^{-1}\right)=1$.

Thus, referring to Theorem 2 , we have in any case that $\tau\left(X Y X Y^{-1}\right)=$ 1, which tells us that $X Y X Y^{-1}$ is elliptic of order 3 . As seen from the general identities (1) and (7), it means that

$$
1=\tau^{2}(Y)+\tau^{2}(X Y)-\tau(X) \tau(Y) \tau(X Y)
$$

or, what is easily seen to be the same

$$
1=\tau^{2}(Y)-\tau(X Y) \tau\left(X^{-1} Y\right) .
$$

Consider the subgroup $H$ generated by $X$ and $Y_{1}=Y X Y^{-1}$. By Theorem 1, also $H$ is extreme. We may as well take $X$ and $Y^{*}=X Y_{1}$ (elliptic of order 3) as generators. Substituting $Y^{*}$ for $Y$ in (10), we obtain

$$
0=\tau\left(X Y^{*}\right) \tau\left(Y_{1}\right)
$$

and since $\tau\left(Y_{1}\right)=\tau(X) \neq 0$, we see that $X Y^{*}$ is elliptic of order 2 .

Since $X$ is either elliptic of order at least 7 or parabolic, we have proved that $H$ is one of the triangle groups spoken of in Theorem 3. But such groups are maximal, that is, such groups cannot be subgroups of strictly larger Fuchsian groups (see Greenberg [1]). Thus $H=G$. 
Using the identity

$$
\begin{aligned}
& {\left[\tau\left(X^{n} Y X^{-n} Y^{-1}\right)-2\right]\left[\tau^{2}(X)-4\right] } \\
= & {\left[\tau\left(X Y X^{-1} Y^{-1}\right)-2\right]\left[\tau^{2}\left(X^{n}\right)-4\right], }
\end{aligned}
$$

it can be shown that

$$
\tau^{2}(X)=4 \cos ^{2} \frac{\pi}{q},
$$

where $q$ is the order of $X$. In fact, if (12) were not true, then we could find a power of $X$ which together with $Y$ would generate a nonelementary and, by (2) and (*), non-discrete group, contradicting the discreteness of $G$.

The triangle groups in question are uniquely determined (up to conjugation) by "canonical generators" with

$$
\left[\tau^{2}(X), \tau^{2}(Y), \tau^{2}(X Y)\right]=\left[4 \cos ^{2} \frac{\pi}{q}, 1,0\right] .
$$

It is a routine matter to check that they are extreme. It concludes the proof of Theorem 3 .

One can show that there are uncountably many non-conjugate, nonFuchsian extreme groups. Some are Kleinian with finite sided fundamental polyhedra while others are non-Kleinian (first kind). In the latter category groups with finite polyhedra (finite volume) as well as groups with infinite polyhedra (first kind degeneracy) can be found. And in spite of the general results (Theorems 1 and 2), there are still infinitely many distinct "extreme signatures."

\section{References}

[1] GreenberG, L.: Maximal Fuchsian groups. - Bull. Amer. Math. Soc. 4, 1963, $569-573$

[2] Jørgensen, T.: On discrete groups of Möbius transformations. - Amer. J. Math. (to appear).

[3] LeHneR, J.: Discontinuous groups and automorphic functions. - Amer. Math. Soc. Mathematical Surveys 8, Providence, Rhode Island, 1964.

University of Minnesota

School of Mathematics

Minneapolis, Minnesota 55455

USA

University of Copenhagen

Department of Mathematics

2100 Copenhagen

Denmark

Received 7 April 1975
University of Helsinki Department of Mathematics SF-00100 Helsinki 10 Finland 